\title{
Validity of Physical Activity Monitors in Assessing Energy Expenditure in Normal, Overweight, and Obese Adults
}

\author{
Ann M. Swartz*, Scott J. Strath, Nora E. Miller, Elizabeth K. Grimm, Lauren A. Ewalt, \\ Michael S. Loy and Keith P. Gennuso
}

Department of Human Movement Sciences, University of Wisconsin-Milwaukee, Milwaukee WI, USA

\begin{abstract}
A comparison of the validity of downloadable motion sensors, which use either a glass-enclosed magnetic reed proximity switch technology, a piezo-electric sensor accelerometer with a horizontal beam technology, or an internal pendulum based mechanism to determine energy expenditure (EE), across different body sizes does not exist. Therefore, the purpose of this study was to determine the validity of three different downloadable motion sensors to estimate EE during walking activity in normal weight, overweight and obese volunteers. Forty-eight participants completed this study. Each participant had their body height and mass measured and completed a treadmill walking protocol. Body mass index (BMI) was calculated. The treadmill walking protocol included six 5-minute stages starting at $1.5 \mathrm{mph}$ and increasing by $0.5 \mathrm{mph}$, up to $4.0 \mathrm{mph}$ while grade was constant at $0 \%$ for the duration of the test. The Kenz Life-Corder EX (LC), the Omron HJ-700IT (OM) and the Sportbrain iStep X1 (SB) were worn during the treadmill walking protocol. Heart rate, oxygen consumption, carbon dioxide production and EE estimated from the motion sensors were monitored throughout the walking protocol. Results showed the OM overestimated net EE in normal, overweight and obese participants. The LC underestimated gross EE in all groups. The SB overestimated net EE in normal BMI participants, was not significantly different from the criterion measure of net EE in overweight participants and underestimated net EE in obese individuals. This study demonstrates that these devices do not offer the accuracy needed to provide precise feedback on EE for individuals with varying BMI levels.
\end{abstract}

Key Words: Pedometer, Walking, Energy Expenditure, Kilocalories.

\section{INTRODUCTION}

Obesity is a major public health concern. Despite efforts to decrease obesity prevalence, the number of obese adults continues to rise. To combat the obesity epidemic, many weight loss programs focus on the energy balance equation, specifically decreasing energy consumed and increasing energy expended. Exercise, and more commonly physical activity (PA) are used to increase energy expenditure (EE). However, a challenge for weight loss interventions is to accurately assess total daily EE from PA.

Motion sensors are commonly used in PA interventions to increase activity level because they are inexpensive, accurate at assessing steps taken in individuals of varying body sizes and provide immediate feedback [1-6]. Although steps per day recommendations for adults are available $(10,000$ steps/d $[7,8]$ or 2,000 steps/d above baseline daily steps [9]), this information may not be meaningful to overweight and obese individuals trying to create a negative energy balance in order to lose weight. To facilitate this need, motion sensors providing caloric feedback may be beneficial and a number of commercially available motion sensors have that capability.

An apparent trend in the technological advancement of motion sensor technology is to automate the downloading of

*Address correspondence to this author at the Department of Human Movement Sciences, The University of Wisconsin-Milwaukee, Enderis Hall Room 453, P.O. Box 413, Milwaukee, WI 53201-0413, USA;

E-mail: aswartz@uwm.edu activity accrued throughout a day, a week, or other period of time. Such automation appears advantageous to users, but also to researchers, removing the reliance on participants to read and document total steps or EE accrued over a specific period of time. If automated or downloadable motion sensors are able to provide accurate information on EE throughout the day, they could be used effectively in weight loss interventions to enhance efficacy and overall compliance.

Some studies have examined downloadable pedometers, such as Crouter et al. [4], who compared two downloadable devices in a normal weight sample of ten individuals. Others have evaluated the Omron pedometer at various treadmill walking speeds $[10,11]$. This device has also been examined for accuracy of steps taken by body placement position in normal weight and obese individuals [12]. To date, a comprehensive study examining the validity of multiple devices which use varied technologies to determine EE, across different body sizes does not exist. Therefore, the purpose of this study was to determine the validity of three different downloadable motion sensors to estimate EE during treadmill walking in normal weight, overweight and obese volunteers.

\section{METHODS}

\section{Participants}

Forty-eight individuals (20 males, 28 females) of varying body size volunteered to participate in this study. All participants were between the ages of 20 and 50 years, were able bodied and were free from diseases, disorders, or orthopedic 
conditions that may impair the participants' ability to walk on a motorized treadmill. All testing took place in a laboratory. The University IRB reviewed and approved the testing protocol.

\section{Study Procedures}

On their single visit to the laboratory, participants completed a health history and demographic questionnaire to identify health issues that may compromise the safety of participants during assessment. Measurements of height, mass, waist circumference and stride length were taken. Finally, a treadmill walking protocol was performed while expired gases were assessed and motion sensors were worn.

\section{Anthropometrics and Stride Length Assessment}

Measurements of height and mass were obtained in duplicate with the participant wearing minimal clothing and no shoes using a calibrated physician's balance beam scale and a stadiometer (Continental Scale Corporation, Bridgeview, IL). Body mass was measured to the nearest $0.01 \mathrm{~kg}$ and height was measured to the nearest $0.1 \mathrm{~cm}$. Body mass index (BMI) was calculated according to the formula body mass $(\mathrm{kg})$ divided by height squared $\left(\mathrm{m}^{2}\right)$. BMI was classified according to the NHLBI Obesity Education Initiative Expert Panel on the Identification, Evaluation, and Treatment of Overweight and Obesity in Adults [13] and the World Health Organization [14] [normal $\left(\leq 24.9 \mathrm{~kg} / \mathrm{m}^{2}\right)$, overweight $\left(25-29.9 \mathrm{~kg} / \mathrm{m}^{2}\right)$, and obese $\left.\left(\geq 30.0 \mathrm{~kg} / \mathrm{m}^{2}\right)\right]$. Waist circumference (narrowest part of the torso between the most inferior rib and the iliac crest) measurements were recorded using a plastic tape fitted with a tension handle. All waist circumference measurements were taken in duplicate to the nearest $0.1 \mathrm{~cm}$ at the end of exhalation, with the average measurement recorded for analysis.

Each participant's stride length was determined using a standardized walking distance. Each participant was asked to walk from a predetermined starting point, beginning with their feet together, to a floor marker $103.6 \mathrm{~m}$ away. The distance between the heel of the foot that crossed the marker and the marker itself was measured and added to the distance walked. Finally, the total distance walked was divided by the number of steps taken to derive stride length.

\section{Treadmill Protocol}

The submaximal treadmill (Trackmaster TMX425C, FullVision Inc, Newton, KS) walking protocol was standardized across all participants. The protocol included six 5 -minute stages starting at $1.5 \mathrm{mph}$ and increasing to 2.0 $\mathrm{mph}, 2.5 \mathrm{mph}, 3.0 \mathrm{mph}, 3.5 \mathrm{mph}$, and $4.0 \mathrm{mph}$ while grade was constant at $0 \%$ for the duration of the test. Heart rate, oxygen consumption, and carbon dioxide production were monitored throughout the exercise test. Measures of heart rate were collected every minute using a Polar (S610i, Polar Electro, Finland) watch and transmission band placed around the chest. Oxygen uptake and carbon dioxide production were measured continuously in expired air, following standardized procedures, via a metabolic measurement system (ParvoMedics True One 2400, Sandy, UT). This metabolic measurement system has been shown to be valid across a wide range of resting to exercise intensities [15]. During the treadmill walking, participants wore the three motion sensors on a belt at waist level.

\section{Motion Sensors}

The Kenz Life-Corder EX (LC; Suzuken Co. Ltd., Nagoya, Japan) was placed on the midline of the right thigh on a belt at the level of the waist during the treadmill protocol. The Omron HJ-700IT (OM; Omron Corporation, Kyoto, Japan) and the Sportbrain iStep X1 (SB: Sportbrain Holdings Inc., Naples, FL) were placed on the left side of the body in the midline of the thigh, on a belt at the level of the waist during the treadmill protocol. Crouter et al. [4] have shown high correlation coefficients $(\mathrm{ICC}=0.83,95 \% \mathrm{CI}=0.71$, 0.90 for OM HJ-105; Kenz Lifecorder ICC $=0.94,95 \%$ $\mathrm{CI}=0.90,0.97)$ between pedometers worn on the left and right side of the body.

\section{Omron HJ-700IT}

The OM pedometer has a glass-enclosed magnetic reed proximity switch that counts steps, "aerobic" steps, net kilocalories expended and distance walked and can store this data for up to seven days. Body mass and stride length are entered into the OM prior to use.

\section{$\underline{\text { Kenz Life-Corder EX }}$}

The LC, based on the Kenz accelerometer, is a piezoelectric sensor accelerometer with a horizontal beam; steps are "determined by the number of zero-crossings on the curve depicting instantaneous acceleration versus time" [16]. Information on gender, age, height, body mass, and the date and time were entered into the LC, which then provides information on steps, daily gross kilocalories expended, daily activity EE, physical activity intensity level (every 4 seconds) and can store up to 200 days of data. Additionally, the LC uses age, gender, height and mass of the individual to calculate basal metabolic rate using a standard formula [17]. Further details on the specifics of the LC can be found in Kumahara et al. [16].

\section{Sportbrain iStep X1}

The SB pedometer has an internal pendulum based mechanism that provides information on the number of steps taken, distance walked and net kilocalories expended. Prior to use, information on height, weight, date of birth, gender and stride length were entered into the device.

\section{Data Analysis}

Gross EE was measured by the metabolic measurement system. Net kilocalories were calculated by subtracting estimated resting EE $(3.5 \mathrm{ml} / \mathrm{kg} / \mathrm{min})$ from measured gross EE. Energy expenditure values from the metabolic measurement system were used as the criterion measure. Energy expenditure from each of the motion sensors was read directly from the motion sensor screen.

Descriptive statistics were run on all variables. Normality of data was assessed using histograms and tests of skew. Mixed between-within subjects analysis of variance was 
performed on difference scores (criterion (indirect calorimetry) $\mathrm{EE}$ - pedometer $\mathrm{EE}$ ) to determine which pedometer was most effective in assessing EE across BMI categories during each treadmill speed. Tukey post hoc tests were performed to determine between group differences. Analyses were performed using SPSS ${ }^{\circledR} 16.0$ for Windows (SPSS Inc., Chicago, IL). The alpha level was set at 0.05 .

\section{RESULTS}

Participants were young to middle-aged individuals (age range 20-50y) with a large variation in BMI (18.6- 36.8 $\mathrm{kg} / \mathrm{m}^{2}$ ). Descriptive data are shown in Table 1. Stride length did not differ between the normal BMI, overweight or obese groups $(p=0.585)$. As expected, measured gross EE increased with walking speeds and differed between groups $(p<0.001$, Table 2). During the data collection sessions, the SB failed to record kilocalories for one participant at stage $1(1.5 \mathrm{mph})$, for two participants during stage $6(4 \mathrm{mph})$ and did not record throughout the walking protocol for one additional participant.

Table 3 shows mean error scores (criterion-device) for energy expended by all participants combined at all treadmill walking speeds. The mean error score for the LC (gross criterion EE- device EE) was significantly different from zero at all speeds except $3.5 \mathrm{mph}$ [1.5 mph $(p<0.001), 2.0$ mph ( $p<0.001), 2.5 \mathrm{mph}(p<0.001), 3.0(p<0.001)$, and 4.0 $(p=0.019)]$. The mean error scores for the OM (net criterion
EE- device EE) were significantly different from zero at speeds of $2.0 \mathrm{mph}(p<0.001), 2.5 \mathrm{mph}(p<0.001), 3.0 \mathrm{mph}$ $(p<0.001)$ and $3.5 \mathrm{mph}(p<0.001)$. The mean error scores for SB (net criterion EE- device EE) were significantly different from zero at $2.5 \mathrm{mph}(p=0.027)$ and $4.0 \mathrm{mph}(p=0.002)$.

\section{Normal BMI}

Results from the mixed between-within analysis of variance showed that the SB was significantly different than the criterion measure of net $\mathrm{EE}$ at $1.5 \mathrm{mph}(\mathrm{p}=0.04), 2.0 \mathrm{mph}$ $(p=0.002), 2.5 \mathrm{mph}(p<0.001), 3.0 \mathrm{mph}(p<0.001)$ and 3.5 $\mathrm{mph}(p=0.013)$, for the normal BMI group. There was a significant difference between OM net EE and criterion net EE at speeds of $2.0 \mathrm{mph}(p<0.001), 2.5 \mathrm{mph}(p<0.001), 3.0$ $(p<0.001)$ and $3.5(p=0.018)$. Finally, the LC estimates of gross EE were significantly different than measured gross $\mathrm{EE}$ in the normal BMI group at speeds of $1.5 \mathrm{mph}(p<0.001)$, $2.0 \mathrm{mph}(p<0.001), 2.5 \mathrm{mph}(p<0.001), 3.0 \mathrm{mph}(p<0.001)$ and $4.0 \mathrm{mph}(p=0.005)$. Fig. (1) shows the percent of actual EE measured by each motion sensor for each participant classified as having a normal BMI.

\section{Overweight BMI}

While walking on the treadmill, estimates of EE were significantly different between the SB and criterion net EE at $4.0 \mathrm{mph}(p=0.001)$ for the overweight BMI group. The OM estimates of net EE were significantly different than the

Table 1. Descriptive Characteristics of Participants (mean \pm SD)

\begin{tabular}{|c|c|c|c|c|}
\hline & $\begin{array}{c}\text { All } \\
(\mathbf{N = 4 8})\end{array}$ & $\begin{array}{c}\text { Normal BMI } \\
(\mathbf{n = 2 5})\end{array}$ & $\begin{array}{c}\text { Overweight } \\
(\mathbf{n = 1 2}) \\
(\mathbf{n = 1 1})\end{array}$ \\
\hline \hline Age $(\mathrm{y})$ & $33.0 \pm 10.7$ & $32.0 \pm 11.0$ & $31.9 \pm 10.5$ & $36.4 \pm 10.4$ \\
\hline Height $(\mathrm{cm})$ & $170.4 \pm 12.6$ & $168.4 \pm 15.0$ & $171.9 \pm 9.3$ & $173.4 \pm 9.5$ \\
\hline Body mass index $\left(\mathrm{kg} / \mathrm{m}^{2}\right)$ & $25.7 \pm 5.1$ & $21.6 \pm 2.0 \dagger$ & $27.3 \pm 1.0^{*}$ & $33.2 \pm 2.1^{*} \dagger$ \\
\hline Waist circumference $(\mathrm{cm})$ & $81.4 \pm 11.9$ & $72.8 \pm 6.4 \dagger$ & $84.5 \pm 4.5^{*}$ & $97.0 \pm 8.8^{*} \dagger$ \\
\hline Stride length $(\mathrm{cm} / \mathrm{step})$ & $72.9 \pm 6.9$ & $72.6 \pm 7.9$ & $74.7 \pm 6.9$ & $71.9 \pm 4.6$ \\
\hline
\end{tabular}

Note. $* p<0.01$, significantly different than normal BMI group; $\dagger p<0.01$, significantly different than overweight BMI group.

Table 2. Measured Gross Energy Expenditure (Kilocalories) During Five Minutes of Treadmill Walking at Various Speeds for Normal, Overweight and Obese Participants (mean \pm SD)

\begin{tabular}{|c|c|c|c|}
\hline & $\begin{array}{c}\text { Normal BMI } \\
(\mathbf{n = 2 5})\end{array}$ & $\begin{array}{c}\text { Overweight } \\
(\boldsymbol{n}=\mathbf{1 2})\end{array}$ & $\begin{array}{c}\text { Obese } \\
(\boldsymbol{n}=\mathbf{1 1})\end{array}$ \\
\hline \hline $1.5 \mathrm{mph}$ & $13.26 \pm 2.65 \dagger$ & $16.21 \pm 2.06^{*}$ & $20.02 \pm 3.19^{* \dagger}$ \\
\hline $2.0 \mathrm{mph}$ & $14.44 \pm 2.24 \dagger$ & $17.63 \pm 1.98^{*}$ & $21.65 \pm 2.80^{* \dagger}$ \\
\hline $2.5 \mathrm{mph}$ & $15.85 \pm 2.01 \dagger$ & $19.92 \pm 2.26^{*}$ & $28.77 \pm 3.21 * \dagger$ \\
\hline $3.0 \mathrm{mph}$ & $20.10 \pm 8.42$ & $23.07 \pm 2.0^{*}$ & $33.80 \pm 3.75 * \dagger$ \\
\hline $3.5 \mathrm{mph}$ & $21.56 \pm 2.83 \dagger$ & $27.31 \pm 2.77^{*}$ & $43.50 \pm 5.31 * \dagger$ \\
\hline $4.0 \mathrm{mph}$ & $26.54 \pm 3.42 \dagger$ & $33.52 \pm 3.58^{*}$ & \\
\hline
\end{tabular}

Note. ${ }^{*} p<0.01$, significantly different than normal BMI group; $\dagger p<0.01$, significantly different than overweight BMI group. 
Table 3. Mean Error Scores (Criterion-Device) for Energy Expended at All Treadmill Walking Speeds

\begin{tabular}{|c|c|c|c|c|c|c|c|c|c|c|c|c|c|c|}
\hline \multirow[b]{3}{*}{$\begin{array}{l}\text { Treadmill } \\
\text { Speed } \\
\text { (mph) }\end{array}$} & \multirow[b]{3}{*}{$\begin{array}{l}\text { Indirect } \\
\text { Calorimetry, } \\
\text { mean } \pm \text { SD } \\
\text { (Gross kcals) }\end{array}$} & \multirow{2}{*}{\multicolumn{4}{|c|}{$\begin{array}{c}\mathrm{t}=0 \\
\text { Life-corder }\end{array}$}} & \multirow[b]{3}{*}{$\begin{array}{l}\text { Indirect } \\
\text { Calorimetry, } \\
\text { mean } \pm \text { SD } \\
\text { (Net kcals) }\end{array}$} & \multicolumn{8}{|c|}{$t=0$} \\
\hline & & & & & & & \multicolumn{4}{|c|}{ Sportbrain } & \multicolumn{4}{|c|}{ Omron HJ-700IT-T54 } \\
\hline & & \begin{tabular}{|l} 
Mean \\
Difference \\
(kcals)
\end{tabular} & $\mathbf{n}$ & $\begin{array}{l}\text { LLOA } \\
\text { (kcals) }\end{array}$ & $\begin{array}{l}\text { ULOA } \\
\text { (kcals) }\end{array}$ & & \begin{tabular}{|l} 
Mean \\
Difference \\
(kcals)
\end{tabular} & n & $\begin{array}{l}\text { LLOA } \\
\text { (kcals) }\end{array}$ & $\begin{array}{l}\text { ULOA } \\
\text { (kcals) }\end{array}$ & \begin{tabular}{|l} 
Mean \\
Difference \\
(kcals)
\end{tabular} & $\mathbf{n}$ & $\begin{array}{l}\text { LLOA } \\
\text { (kcals) }\end{array}$ & $\begin{array}{l}\text { ULOA } \\
\text { (kcals) }\end{array}$ \\
\hline 1.5 & $15.1 \pm 3.4$ & $7.8 * *$ & 48 & 6.0 & 9.6 & $9.4 \pm 2.8$ & -0.5 & 46 & -2.7 & 1.7 & -0.9 & 48 & -3.6 & 1.8 \\
\hline 2.0 & $16.6 \pm 3.5$ & $3.7 * *$ & 48 & 2.8 & 4.6 & $10.7 \pm 3.1$ & -1.7 & 47 & -4.3 & 0.9 & $-6.4 * *$ & 48 & -7.5 & -5.4 \\
\hline 2.5 & $18.3 \pm 3.6$ & $3.2 * *$ & 48 & 2.5 & 3.9 & $12.2 \pm 4.2$ & $-2.3^{*}$ & 47 & -4.3 & -0.3 & $-7.0 * *$ & 48 & -8.6 & -5.4 \\
\hline 3.0 & $22.4 \pm 7.1$ & $4.3 * *$ & 48 & 2.6 & 6.1 & $15.8 \pm 4.9$ & 0.3 & 47 & -1.6 & 2.1 & $-4.8 * *$ & 48 & -6.2 & -3.4 \\
\hline 3.5 & $25.4 \pm 5.4$ & 1.6 & 48 & -0.4 & 3.6 & $19.9 \pm 6.4$ & 1.3 & 47 & -1.8 & 4.3 & $-5.2 * *$ & 48 & -8.0 & -2.4 \\
\hline 4.0 & $31.4 \pm 7.0$ & $3.0 *$ & 48 & 0.5 & 5.5 & $26.0 \pm 9.2$ & $5.4 * *$ & 45 & 2.2 & 8.6 & 0.9 & 48 & -2.7 & 4.5 \\
\hline
\end{tabular}

Note. LLOA, lower limits of agreement; ULOA, upper limits of agreement; ${ }^{*} p<0.05 ; * * p<0.01$.

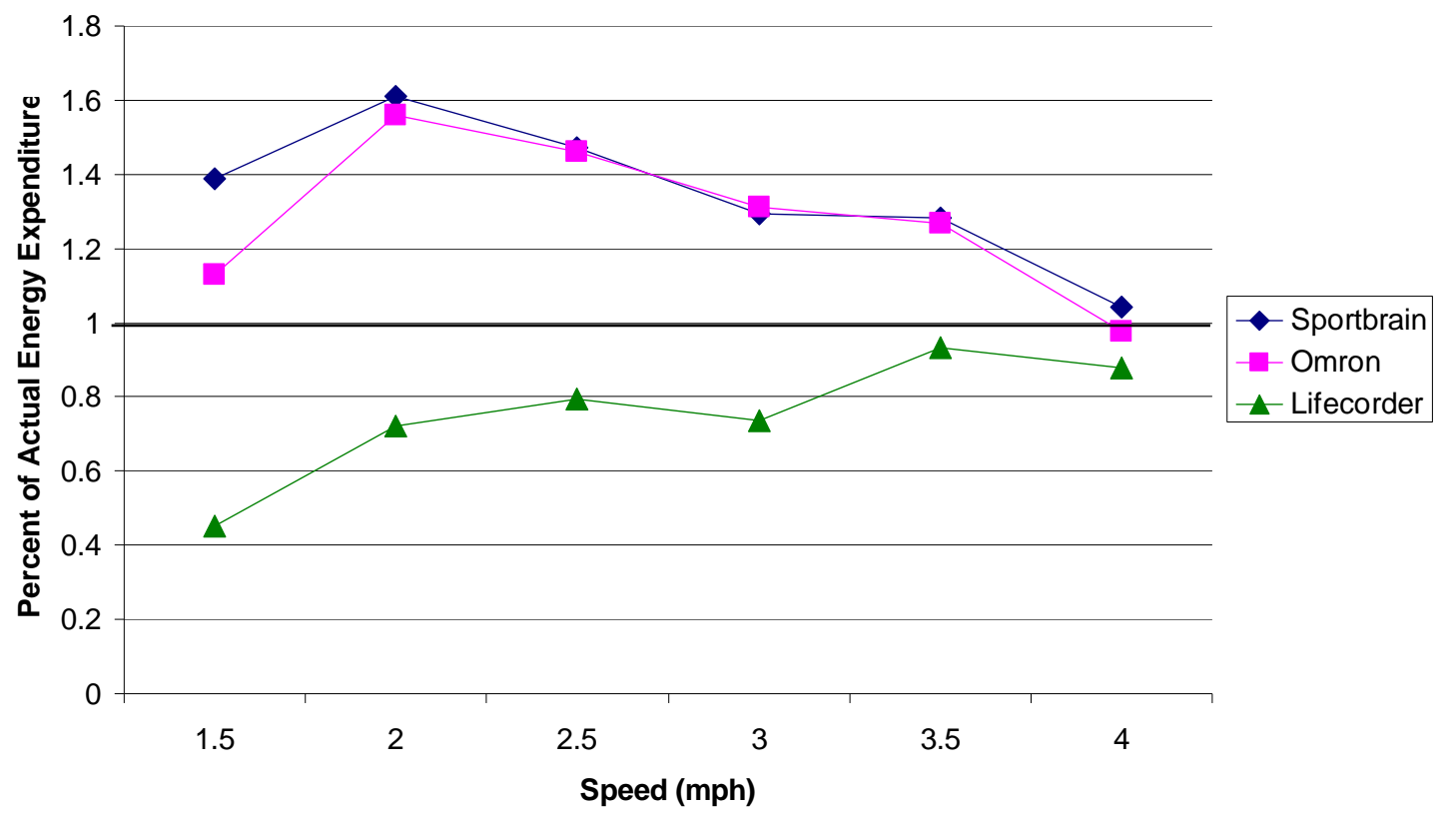

Note. OM and SB are displayed in percent of actual net EE. LC is displayed in percent of actual gross EE. SB $n=24 ;$ OM $n=25, \mathrm{LC}, n=25$.

Fig. (1). Percent of actual kilocalories for participants with a normal BMI across treadmill walking speeds.

criterion measured EE at speeds of $2.0 \mathrm{mph}(p<0.001), 2.5$ $\mathrm{mph}(p<0.001), 3.0 \mathrm{mph}(p<0.001)$, and $3.5 \mathrm{mph}(p<0.001)$. Finally, the LC was significantly different than criterion for gross EE at speeds of $1.5 \mathrm{mph}(p<0.001), 2.0 \mathrm{mph}(p=0.014)$, $2.5 \mathrm{mph}(p<0.001), 3.0 \mathrm{mph}(p=0.03)$, and $4.0 \mathrm{mph}$ $(p=0.007)$ in the overweight BMI group. Fig. (2) shows the percent of actual EE measured by each motion sensor for the participant classified as having an overweight BMI.

\section{Obese BMI}

Results showed a significant difference in SB estimated net $\mathrm{EE}$ and criterion measured net $\mathrm{EE}$ at speeds of $1.5 \mathrm{mph}$ $(p=0.039), 3.0 \mathrm{mph}(p<0.001), 3.5 \mathrm{mph}(p<0.001)$ and 4.0 mph $(p<0.001)$. The OM estimates of EE were significantly different than criterion measured $\mathrm{EE}$ at speeds of $2.0 \mathrm{mph}$ $(p<0.001), 2.5 \mathrm{mph}(p=0.002), 3.0(p=0.024)$ and $4.0 \mathrm{mph}$ $(p=0.001)$. Finally, gross EE estimated by the LC was significantly different than gross $\mathrm{EE}$ measured by the criterion method at all speeds except $3.0 \mathrm{mph}[1.5 \mathrm{mph}$ $(p<0.001), 2.0 \mathrm{mph}(p<0.001), 2.5 \mathrm{mph}(p<0.001), 3.5 \mathrm{mph}$ $(p=0.032)$, and $4.0 \mathrm{mph}(p=0.010)]$ for the obese group. Fig. (3) shows the percent of actual EE measured by each motion sensor for the participant classified as having an obese BMI.

In general, the OM overestimated net $\mathrm{EE}$ in normal, overweight and obese participants, the LC underestimated gross $\mathrm{EE}$ in all groups and the SB overestimated net EE in normal BMI participants, was not significantly different from indirect calorimetry in overweight participants and underestimated net EE in obese individuals (Table 4). 


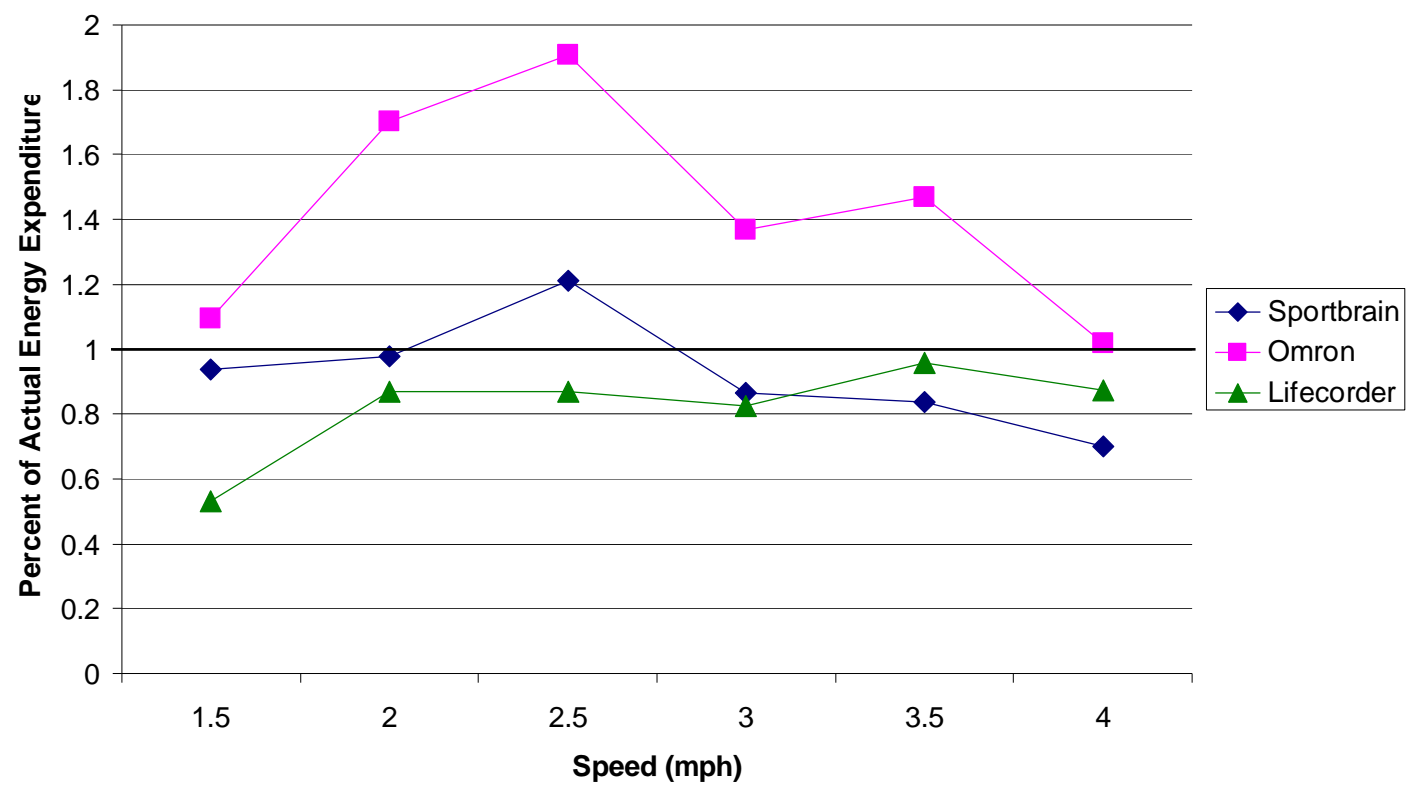

Note. OM and SB are displayed in percent of actual net EE. LC is displayed in percent of actual gross EE. $n=12$.

Fig. (2). Percent of actual kilocalories for overweight participants across treadmill walking speeds.

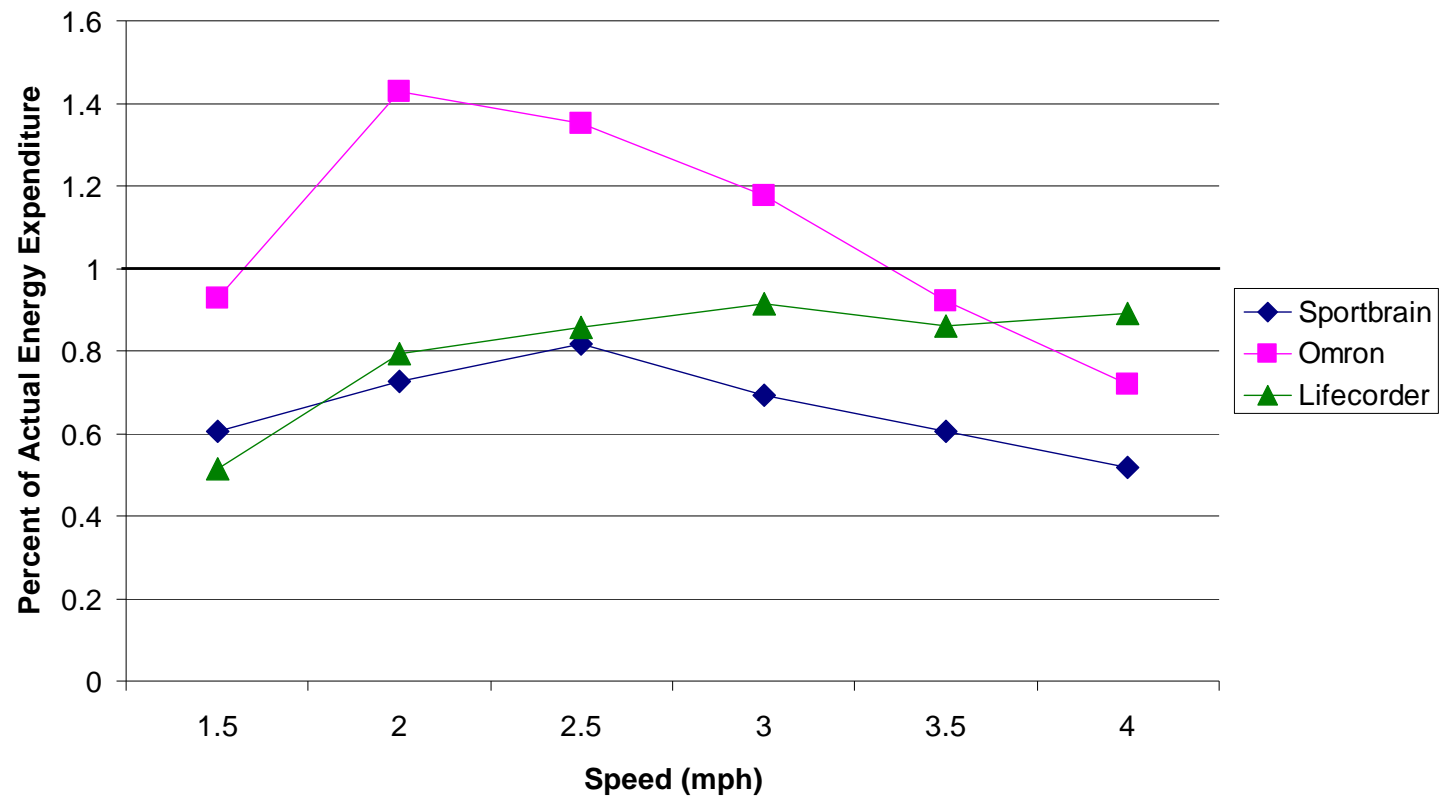

Note. OM and SB are displayed in percent of actual net EE. LC is displayed in percent of actual gross EE. SB $n=9 ;$ OM $n=11, \mathrm{LC}, n=11$.

Fig. (3). Percent of actual kilocalories for obese participants across treadmill walking speeds.

Table 4. Classification of Motion Sensor Accuracy for Estimating Energy Expenditure in Normal BMI, Overweight or Obese Individuals

\begin{tabular}{|c|c|c|c|}
\hline & Overestimation of Measured EE & No Significant Difference from Measured EE & Underestimation of Measured EE \\
\hline \hline Normal BMI $(\mathrm{n}=25)$ & SB, OM & SB & LC \\
\hline Overweight $(\mathrm{n}=12)$ & OM & LC \\
\hline Obese $(\mathrm{n}=11)$ & OM & SB, LC \\
\hline
\end{tabular}

Note: SB data was collected on 24 normal BMI and 9 obese participants. 


\section{DISCUSSION}

Physical activity interventions are commonly used in weight loss and weight maintenance programs to increase $\mathrm{EE}$ and thereby aid in weight loss or weight maintenance. However, estimating EE from PA outside a laboratory setting is difficult. Therefore, a device that can accurately estimate EE from ambulatory activities may help in weight maintenance or weight loss interventions. The primary aim of this study was to assess whether three commonly used downloadable pedometers could accurately estimate EE in individuals of normal, overweight and obese BMI. In general, none of the motion sensors evaluated in this study assessed EE accurately for the normal BMI or obese BMI groups. In the overweight group, the SB did not significantly differ from measured EE except during the fastest walking speed (4 mph). Overall, all motion sensors improved accuracy of estimating EE as walking speed increased (Figs. 1-3). Improved accuracy at higher speeds is most likely due to an increase in accuracy of counting steps at higher speeds, as demonstrated in previous research [4].

Crouter et al. (2003) evaluated earlier versions of the OM (OM HJ-105) and LC (Kenz Lifecorder) in 10 young $(33 \pm 12 \mathrm{y})$ normal to overweight $\left(25.7 \pm \mathrm{kg} / \mathrm{m}^{2}\right)$ individuals, showing the OM HJ-105 overestimated net kilocalories at 2.0, 2.5, 3.0 and $3.5 \mathrm{mph}$, while the Kenz Lifecorder overestimated gross $\mathrm{EE}$ at speeds of 3.0 and $3.5 \mathrm{mph}$. Results from the current study showed that none of the pedometers accurately assessed EE at all walking speeds for the group as a whole. The OM showed similar results to previous research [4], significantly overestimating net kilocalories by $26-60 \%$ at $2.0,2.5,3.0$ and $3.5 \mathrm{mph}$ (Table 2), but was not significantly different than measured EE at $4.0 \mathrm{mph}$. The LC significantly underestimated gross kilocalories by $19 \%$ at 3.0 mph (10-52\% underestimation at 1.5, 2.0, 2.5 and $4.0 \mathrm{mph}$ ), but did not differ from measured $\mathrm{EE}$ at $3.5 \mathrm{mph}$. In general, the SB appeared to most accurately assess EE across BMI at all walking speeds except 2.5 (19\% overestimation) and 4.0 $\mathrm{mph}$ (21\% underestimation). In particular, the SB overestimated $\mathrm{EE}$ in the normal BMI group, underestimated EE in the obese group, and was not significantly different than the criterion measure in the overweight group at speed of 3.5 mph or less.

Previous research has shown piezo-electric pedometers (New Lifestyles NL-2000) to be more accurate at assessing steps taken than spring suspended lever arm pedometers (Digiwalker SW-200) in overweight and obese adults and at slower walking speeds [3]. Crouter et al. concluded increased accuracy could be due to factors such as tilt of the pedometer, waist circumference and/or BMI, which do not appear to impact the piezo-electric pedometers as much as the spring suspended lever arm pedometers [3]. Results from this study, although focusing on EE rather than steps as an outcome, showed the spring suspended lever arm pedometer (SB) estimated EE accurately at more speeds for the overweight group and for the entire group (normal, overweight, obese together) than the accelerometer based motion sensor (KZ) or the glass enclosed magnetic reed proximity switch pedometer (OM).

Inaccurate $\mathrm{EE}$ estimates from the LC for all BMI groups could be explained by the methods of estimating EE. The LC calculates total gross EE based on thermic effect of food, physical activity EE, and an estimation of basal metabolic rate developed on Japanese participants. Kumahara et al. (2004) showed that $66 \%$ of the variance in $24 \mathrm{~h}$ estimates of EE in 71 healthy, lean Japanese males and females was attributed to estimates of basal metabolic rate, suggesting that a large amount of error in the estimate of EE is from the calculation of basal metabolic rate [16].

Information on the methods (treadmill or incidental activity) and participants used to develop the formulas used to estimate EE in each of these devices is not known. However, having this information may help to better understand why some motion sensors underestimate EE and some overestimate EE. Further, it may help improve the accuracy with which these devices estimate EE.

Obesity is a worldwide epidemic impacting health and quality of life. Therefore, efforts to decrease obesity are important. Many weight loss interventions focus on creating a negative energy balance, where an individual expends more energy than is consumed. Motion sensors with the ability to estimate energy expenditure have the potential to aid individuals in creating a negative energy balance. However, results from this study show these devices do not offer the accuracy needed to provide precise feedback on EE for individuals with varying BMI levels. Therefore, other methods including relying on steps taken per day may provide more beneficial feedback on PA level rather than kilocalories expended.

\section{ACKNOWLEDGMENTS}

This work was partially supported by funding received from a Career Development Award from the National Institute on Aging (K01AG025962) and a Center Scientist Award from the Center for Urban Population and Health.

\section{DISCLAIMER}

The results of the present study do not constitute endorsement by the authors of the products described in this paper.

\section{REFERENCES}

[1] Bassett DR, Jr, Ainsworth BE, Leggett SR, et al. Accuracy of five electronic pedometers for measuring distance walked. Med Sci Sports Exerc 1996; 28: 1071-7.

[2] Bassett DR, Jr, Ainsworth BE, Swartz AM, Strath SJ, O'Brien WL, King GA. Validity of four motion sensors in measuring moderate intensity physical activity. Med Sci Sports Exerc 2000; 32: S47180.

[3] Crouter S, Schneider P, Bassett D, Jr. Spring-levered versus piezoelectric pedometer accuracy in overweight and obese adults. Med Sci Sports Exerc 2005; 37: 1673-9.

[4] Crouter S, Schneider P, Karabulut M, Bassett D, Jr. Validity of 10 electronic pedometers for measuring steps, distance, and energy cost. Med Sci Sports Exerc 2003; 35: 1455-60.

[5] Schneider P, Crouter S, Bassett D, Jr. Pedometer measures of freeliving physical activity: comparison of 13 models. Med Sci Sports Exerc 2004; 36: 331-5.

[6] Swartz AM, Bassett DR, Jr., Moore JB, Thompson DL, Strath SJ. Effects of body mass index on the accuracy of an electronic pedometer. Int J Sports Med 2003; 24: 588-92.

[7] Bassett DR, Jr, Strath SJ. Physical Activity Assessments for Health-Related Research. In: Welk G, Dale D, Eds. Human Kinetics. Champaign: IL 2002; pp. 163-77.

[8] Hatano Y. The use of the pedometer for promoting daily walking exercise. J ICHPER-SD 1993; 29: 4-8.

[9] Hill JO, Wyatt HR, Reed GW, Peters JC. Obesity and the environment: where do we go from here? Science 2003; 299: 853-5. 
[10] Hasson RE, Pober DM, Freedson PS. Evaluation of the Omron HJ112 and Yamax Digiwalker SW-701 pedometers during variable speed walking. In: Proceedings of the Walking for Health: Measurement and Research Issues and Challenges; 2005: UrbanaChampaign: ACSM-UIUC Kinesmetrics Symposium Series 2005; p. 26.

[11] Haller J, Hasson RE, Pober DM, Freedson PS. Validation of the Omron HJ-112 pedometer at various walking speeds. In: Proceedings of the Walking for Health: Measurement and Research Issues and Challenges; 2005: Urbana-Champaign: ACSM-UIUC Kinesmetrics Symposium Series 2005; p. 26.

[12] Roberts DE, Hasson RE, Pober DM, Freedson PS. Effect of pedometer position on step count accuracy in normal weight and obese individuals. In: Proceedings of the Walking for Health: Measurement and Research Issues and Challenges; 2005: UrbanaChampaign: ACSM-UIUC Kinesmetrics Symposium Series 2005; p. 34 .
[13] Expert Panel on the Identification Evaluation and Treatment of Overweight in Adults. Clinical guidelines on the identification, evaluation, and treatment of overweight and obesity in adults: Executive summary. Am J Clin Nutr 1998; 68: 899-917.

[14] World Health Organization. Obesity: preventing and managing the global epidemic. Geneva: World Health Organization 2000.

[15] Bassett DR, Jr, Howley ET, Thompson DL, et al. Validity of inspiratory and expiratory methods of measuring gas exchange with a computerized system. J Appl Physiol 2001; 91: 218-24.

[16] Kumahara H, Schutz Y, Ayabe M, et al. The use of uniaxial accelerometry for the assessment of physical-activity-related energy expenditure: a validation study against whole-body indirect calorimetry. Br J Nutr 2004; 91: 235-43.

[17] Health Promotion and Nutrition Division HSB, Ministry of Health and Welfare. Recommended Dietary Allowances for the Japanese. Tokyo: Dai-ichi shuppan Co. Ltd. 1996.

(c) Swartz et al.; Licensee Bentham Open.

This is an open access article licensed under the terms of the Creative Commons Attribution Non-Commercial License (http://creativecommons.org/licenses/by-nc/3.0/) which permits unrestricted, non-commercial use, distribution and reproduction in any medium, provided the work is properly cited. 